\title{
Bipolar pulsed excitation of erbium-doped nanosilicon light emitting diodes
}

\author{
A. Anopchenko, ${ }^{1, a)}$ A. Tengattini, ${ }^{1}$ A. Marconi, ${ }^{1}$ N. Prtljaga, ${ }^{1}$ J. M. Ramírez, ${ }^{2}$ O. Jambois, ${ }^{2}$ \\ Y. Berencén, ${ }^{2}$ D. Navarro-Urrios, ${ }^{2, b)}$ B. Garrido, ${ }^{2}$ F. Milesi, ${ }^{3}$ J.-P. Colonna, ${ }^{3}$ J.-M. Fedeli, ${ }^{3}$ \\ and L. Pavesi ${ }^{1}$ \\ ${ }^{1}$ Nanoscience Laboratory, Department of Physics, University of Trento, Via Sommarive 14, Povo 38123, Italy \\ ${ }^{2}$ Department of Electronics, University of Barcelona, Carrer Martì i Franquès 1, Barcelona 08028, Spain \\ ${ }^{3}$ CEA/Léti, Minatec, 17 rue des Martyrs, 38054 Grenoble cedex 9, France
}

(Received 13 October 2011; accepted 17 February 2012; published online 21 March 2012)

\begin{abstract}
High quantum efficiency erbium doped silicon nanocluster (Si-NC:Er) light emitting diodes (LEDs) were grown by low-pressure chemical vapor deposition (LPCVD) in a complementary metal-oxide-semiconductor (CMOS) line. Erbium (Er) excitation mechanisms under direct current (DC) and bipolar pulsed electrical injection were studied in a broad range of excitation voltages and frequencies. Under DC excitation, Fowler-Nordheim tunneling of electrons is mediated by Er-related trap states and electroluminescence originates from impact excitation of Er ions. When the bipolar pulsed electrical injection is used, the electron transport and Er excitation mechanism change. Sequential injection of electrons and holes into silicon nanoclusters takes place and nonradiative energy transfer to Er ions is observed. This mechanism occurs in a range of lower driving voltages than those observed in DC and injection frequencies higher than the Er emission rate. (C) 2012 American Institute of Physics. [http://dx.doi.org/10.1063/1.3694680]
\end{abstract}

\section{INTRODUCTION}

The Er-doped silicon nanocluster (Si-NC:Er) system is studied as a gain medium in silicon photonics since it could potentially allow for efficient and integrable light sources. ${ }^{1,2}$ Room temperature Si-NC:Er light emitting diodes (LEDs) with an external quantum efficiency (EQE) at $1.54 \mu \mathrm{m}$ larger than $0.1 \%$ have been demonstrated. ${ }^{1,3-5}$ Er excitation in the Si-NC:Er system is either due to a direct impact of hot electrons or via an indirect energy transfer from nonradiative recombination of electron-hole pairs/excitons confined in silicon nanoclusters. The energy transfer is well documented and several mechanisms of the energy transfer under optical excitation have been suggested in recent publications. ${ }^{6-8}$

Pulsed electrical excitation of the Si-NC:Er system has not been studied in as much detail as optical excitation or direct current electroluminescence. Walters et al. ${ }^{9}$ and Peralvarez et al. ${ }^{10}$ demonstrated sequential injection of electrons and holes into Si nanocrystals under a bipolar pulsed excitation of a field-effect LED. Priolo et al. suggested this excitation scheme as a solution to overcome nonradiative Auger deexcitation of Er in Si-NC:Er LEDs. ${ }^{11}$ Miller et al. calculated a modal gain of $2 \mathrm{~dB} / \mathrm{cm}$ in a slot waveguide confined $\mathrm{Si}-\mathrm{NC}: \mathrm{Er}$ under the pulsed excitation which mitigates excited carrier absorption. ${ }^{12}$

In this work, we will evaluate erbium excitation mechanisms and emission in Si-NC:Er LED under electrical pumping using both direct current and bipolar pulsed excitation schemes, i.e., when the polarity of the applied voltage pulse is periodically changed.

\footnotetext{
${ }^{a}$ Electronic mail: anopchenko@science.unitn.it.

b) Present address: Catalan Institute of Nanotechnology (CIN2-CSIC), Campus UAB, Edifici CM3, 08193 Bellaterra, Spain.
}

\section{EXPERIMENTAL}

The silicon nanoclusters (Si-NCs) are formed during 14(1/2)-h-long high temperature anneal at $900{ }^{\circ} \mathrm{C}$ of a silicon-rich silicon oxide layer with a nominal $12 \%$ of silicon excess ( $9 \%$ measured by x-ray photoelectron spectroscopy) grown by low-pressure chemical vapor deposition process (LPCVD). ${ }^{13}$ As measured by secondary ion mass spectrometry, peak Er-ion concentration of $\sim 4 \times 10^{20} \mathrm{~cm}^{-3}$ is obtained by Er implantation with a fluence of $10^{15}$ ions $/ \mathrm{cm}^{2}$ and energy of $25 \mathrm{keV}$. Post-implantation anneal is performed at $800^{\circ} \mathrm{C}$ for $6 \mathrm{~h}$. A schematic layout of the $n$-type complementary metal-oxide-semiconductor (CMOS) Si-NC:Er LED is shown in Fig. 1. The thickness of the active layer is $50 \mathrm{~nm}$. A semitransparent window is formed by a conductive 150nm-thick polycrystalline silicon layer and an antireflection coating of $93 \mathrm{~nm}$ of $\mathrm{Si}_{3} \mathrm{~N}_{4}$ and $136 \mathrm{~nm}$ of $\mathrm{SiO}_{2}$. The device studied in this work has a square gate area with a size of $500 \mu \mathrm{m} \times 500 \mu \mathrm{m}$ and an $n$-type polysilicon gate. The direct current (DC) bias polarity convention is shown in Fig. 1.

Electrical and optical measurements are performed at room temperature. Current-voltage $(I-V)$ characteristics are recorded with an Agilent B1500 A semiconductor device analyzer. High frequency, $100 \mathrm{kHz}$, capacitance-voltage $(C-V)$ measurements are performed with HP 4284 A precision LCR meter. A 2-m-long extension cable is used. The open circuit corrections are performed according to the operation manual. The alternating current (AC) signal voltage level is $50 \mathrm{mV}$. A function generator, Tektronix AFG 3252, coupled with a high-voltage amplifier, Falco Systems WMA-300, is used to drive the device in time-resolved electroluminescence (EL) measurements.

Time-resolved EL signal is collected with a singlephoton detector module, id-Quantique 201, and recorded with a multichannel scaler, SRS 430. EL spectra are collected 


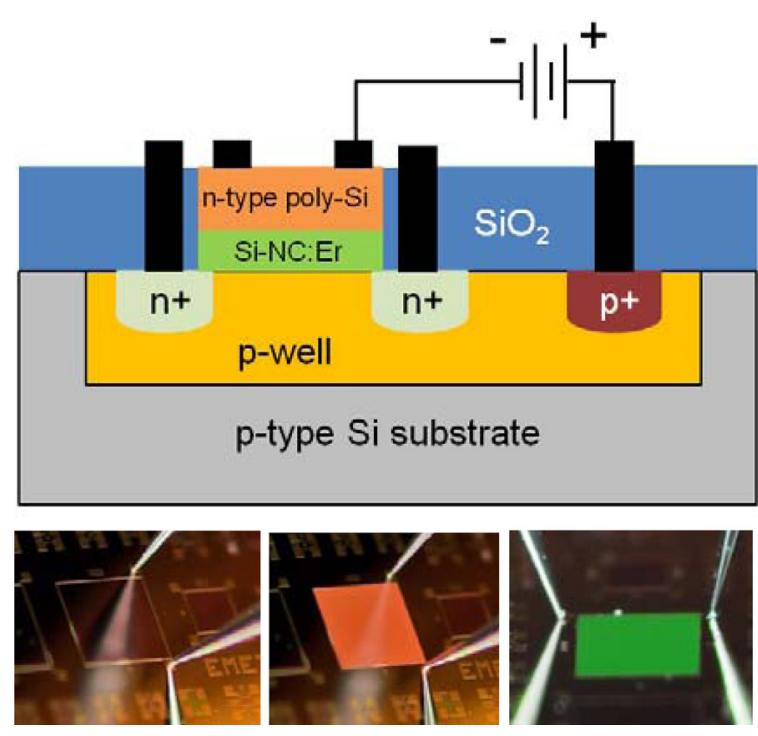

FIG. 1. (top) A schematic cross section of the $n$-type CMOS Si-NC:Er LED layout. Green color stands for the Er-doped Si-NC layer, blue - oxide, and black - titanium-aluminum-copper metal contact sandwich. (bottom, left to right) Photographs of the unbiased Si-NC LED, an orange emitting Si-NC LED under forward bias (a red square at the probe tips) and green emitting Si-NC:Er LED.

using a fiber bundle and analyzed with a Spectra-Pro 2300i monochromator coupled with nitrogen cooled charge-coupled device (CCD) cameras (one in visible and one in infrared, IR). Emitted optical power and EQE of the Si-NC:Er LED are measured using either a calibrated LED or a Ge photodiode. The acceptance angle of the photodiode is taken into account. The photodiode is placed within a few millimeters above the LED. The optical power emitted by the LED is estimated as $P=P_{m} / \sin ^{2} \varphi$, where $\varphi$ is the acceptance angle of the photodiode and $P_{m}$ is a measured fraction of the total optical power emitted into air.

\section{RESULTS AND DISCUSSION}

\section{A. Direct current excitation}

Electrical charge transport in our Si-NC LEDs is due to electric field-enhanced tunneling of electrons with the involvement of either defects ${ }^{14-16}$ or confined energy states of Si-NCs. ${ }^{17,18}$ Erbium implantation produces deep energy trapping levels which change the transport properties of the $\mathrm{Si}$-NC LED. ${ }^{11,19}$ This is supported by the DC $I-V$ and $C-V$ characteristics shown in Fig. 2. The $I-V$ curves of both Si$\mathrm{NC}$ and Er-doped Si-NC LEDs are shown in a voltage range where EL signal is observed under forward bias ( $\mathrm{Si}$ substrate is in accumulation, see Fig. 1). EL emission is also observed under reverse bias in both undoped and doped Si-NC LEDs, but at higher voltages and with lower quantum efficiency. The $I-V$ curves are well described by the Fowler-Nordheim field-enhanced tunneling law ${ }^{17}$ with effective energy barrier heights of 1.4 and $1.9 \mathrm{eV}$ for Si-NC and Si-NC:Er LED, respectively (assuming an effective electron mass of 0.3 $\left.\mathrm{m}_{0}\right) .{ }^{20}$ The Er-doped device is less conductive than the undoped device, which we ascribe to charge trapping at deep energy levels due to Er ion implantation. ${ }^{21}$ This is also supported by the $C-V$ measurements (Fig. 2). A hysteresis loop
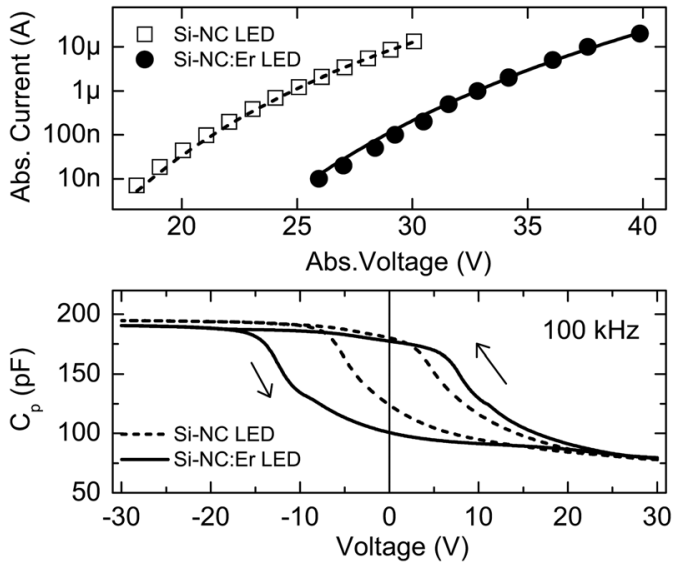

FIG. 2. (top) Forward current-voltage characteristics of Si-NC and Si-NC:Er LEDs. Symbols are experimental data values at which EL is observed, lines are fits to the Fowler-Nordheim tunneling law. (bottom) Capacitance-voltage characteristics of the Er-doped and undoped Si-NC LEDs. The signal frequency is $100 \mathrm{kHz}$. The arrows show bias scanning direction in the $C-V$ measurements.

is observed in the $C-V$ curves, which is due to charge trapping. The hysteresis is wider for the Er-doped Si-NC LED than for the undoped Si-NC LED. Trapped charge density estimated from $C-V$ hysteresis width of the Si-NC LED is $4.3 \times 10^{12} \mathrm{~cm}^{-2}$. Assuming one trapped electron per Si-NC, this value serves as a good estimate of the Si-NC density. ${ }^{22}$

Figure 3 (top panel) shows the integrated spectral EL intensity of the Si-NC and Si-NC:Er LEDs as a function of injection DC current in the visible range and at wavelengths bracketing the $1.54 \mu \mathrm{m}$ Er emission, respectively. For low injection currents, EL intensity at $1.54 \mu \mathrm{m}$ increases linearly with the DC current. However, at high currents a sublinear growth with injected current is observed. On the contrary, the visible emission from $\mathrm{Si}-\mathrm{NCs}$ increases almost linearly (with a slope of $0.91 \pm 0.01$ in the log-log coordinates) as a
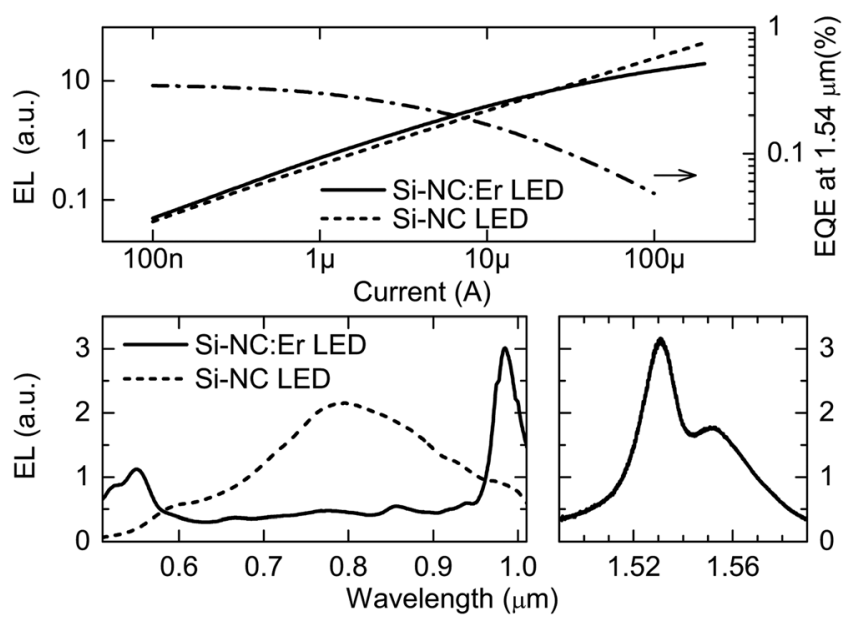

FIG. 3. Integrated EL spectral intensity with wavelengths bracketing 1.54 $\mu \mathrm{m}$ (left axis, solid line) and in the visible range (left axis, dot line) for SiNC:Er LED and Si-NC LED, respectively, as a function of DC injected current. Please note that absolute values of the EL intensity in the visible and IR ranges are not to compare (the $y$-axes are not the same). The dash-dot line shows the corresponding EQE values at $1.54 \mu \mathrm{m}$ (right axis). (bottom) EL spectra at the injected current of $2 \mu \mathrm{A}$. The spectra are normalized to the detection system response. 


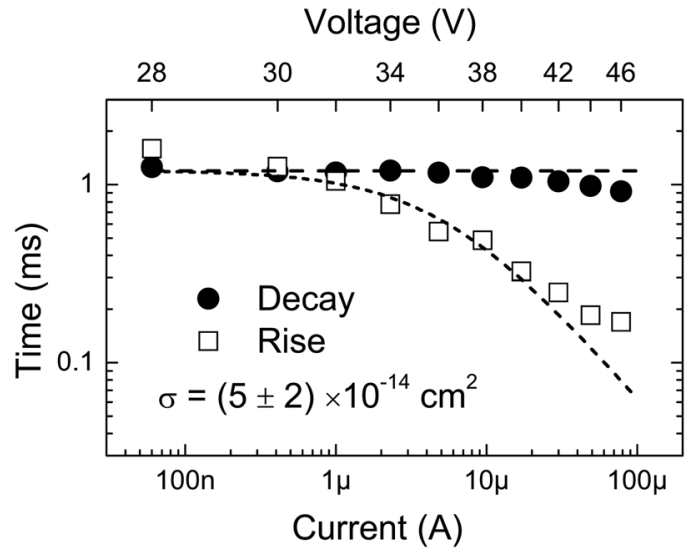

FIG. 4. Exponential decay and rise times of an integrated EL spectral intensity in IR as a function of LED driving voltage (top $x$-axis) and the corresponding current taken from the DC $I-V \mathrm{~s}$ in Fig. 2 (bottom $x$-axis). Measurement uncertainty is smaller than symbol sizes. The Er excitation cross-section value estimated from the data is $(5 \pm 2) \times 10^{-14} \mathrm{~cm}^{2}$. The lines are guides to the eye with a constant value of the decay time of $1.2 \mathrm{~ms}$ and an Er excitation cross-section value of $5.9 \times 10^{-14} \mathrm{~cm}^{2}$.

function of the current. The saturation of the $1.54 \mu \mathrm{m}$ emission may be attributed to both a limited amount of optically active Er ions and to the onset of nonradiative recombination processes. ${ }^{11,16,23}$ The presence of the last is evidenced by the decrease of the luminescence decay time shown in Fig. 4, which we will discuss later.

Figure 3 also shows the EQE of Er-doped Si-NC LED emitting at $1.54 \mu \mathrm{m}$. It is noteworthy that these EQE values are among the best values reported so far for the Er-doped silicon LED (see for example Refs. 24 and 25). The EL spectra of the Si-NC LED and of the Si-NC:Er LED for a same injected current of $2 \mu \mathrm{A}$ are shown in the bottom panel of the Fig. 3. The emission of the Si-NC LED is characterized by a broad peak centered at around $770 \mathrm{~nm}$ (see the middle picture of the low panel of Fig. 1), which originates from excitonic recombinations in the Si-NCs. The Si-NC:Er LED emission spectrum shows in addition to the broad $\mathrm{Si}-\mathrm{NC}$ emission several sharp peaks at around 550, 660, 850, 980, and $1535 \mathrm{~nm}$ due to the excited Er states emission (presence of the peaks at 660 and $850 \mathrm{~nm}$ is more evident at higher currents). It has to be noted that the green emission at $550 \mathrm{~nm}$ (see the right picture of the low panel of Fig. 1) is not due to the Er up-conversion because the peak intensities of the various emission bands increase linearly as the injection current increases. The linear increase is observed in a broad range of currents up to $20 \mu \mathrm{A}$. At larger currents, we observed some signs of the cooperative up-conversion. ${ }^{26}$ High driving voltages (Fig. 2) along with the presence of the multiple Er peaks indicate that $\mathrm{Er}$ emission is mainly due to direct impact excitation of Er ions and not to indirect Er excitation via energy transfer from Si-NCs. The energy transfer between $\mathrm{Si}$-NCs and Er ions cannot be completely ruled out since the $\mathrm{Si}-\mathrm{NC}$ emission peak is much weaker in the Si$\mathrm{NC}$ :Er LED than in the undoped device.

Another argument for the interaction between $\mathrm{Er}$ and $\mathrm{Si}$ NCs is the value of the excitation cross-section of Er. This can be estimated by measuring the exponential rise and decay time of EL. ${ }^{4,11,23}$ The result is shown in Fig. 4. The excitation cross-section value extracted from the data is $(5 \pm 2) \times 10^{-14} \mathrm{~cm}^{2}$. Note that the evaluation is based only on the rise and decay times for driving voltages larger than $30 \mathrm{~V}$. For lower driving voltages, the $1.54-\mu \mathrm{m}$ EL rise time exceeds $1.20 \pm 0.02 \mathrm{~ms}$, which is equal to the EL decay time. We also note that the EL decay time is similar to the measured 1.54- $\mu \mathrm{m}$ photoluminescence (PL) lifetime of $1.31 \pm 0.05 \mathrm{~ms}$. We measured an excitation cross-section value which is larger than the known value of direct impact excitation of $\mathrm{Er}$ in $\mathrm{SiO}_{2},(6 \pm 2) \times 10^{-15} \mathrm{~cm}^{2},{ }^{27}$ and which is close to the indirect Er excitation cross-section value for $\mathrm{Er}$ coupled to $\mathrm{Si}-\mathrm{NCs}{ }^{4,25}$ It is noteworthy that the decrease in the decay lifetime only moderately accounts for the decrease in EQE shown in Fig. 3. Saturation of optically active Er concentration might account for the rest of this decrease.

In summary, the results of the DC excitation presented in this section suggest that electrical current is due to electron tunneling mediated by Si-NCs in the Si-NC LED and Er-related defects in the Si-NC:Er LED. They also show that $\mathrm{Er}$ is primarily excited by impact of high energy electrons. The impact of holes is believed to be small because of a negligible hole current due to the larger valence band offset of $\mathrm{Si}$ and $\mathrm{Si}-\mathrm{NCs}$ than the conduction band offset. ${ }^{28}$

\section{B. Bipolar pulsed excitation}

Figure 5 shows the peak EL intensity at $770 \mathrm{~nm}$ and the spectrally integrated EL intensity bracketing $1.54 \mu \mathrm{m}$ as a function of the driving frequency, $f$, for a bipolar pulsed excitation scheme. Here the LEDs are driven by varying the bias periodically and rapidly (within $\sim 400 \mathrm{~ns}$ ) from forward to reverse and from reverse to forward with a square waveform at a frequency $f$. At low driving frequencies, $f \ll 1 \mathrm{kHz}$, the EL intensity at $1.54 \mu \mathrm{m}$ (bottom panel in Fig. 5) decreases a little with the frequencies for high driving voltages and increases for low driving voltages, being much weaker for the low bias. As the driving frequency approaches $800 \mathrm{~Hz}$, which corresponds to the inverse of Er emission lifetime, the

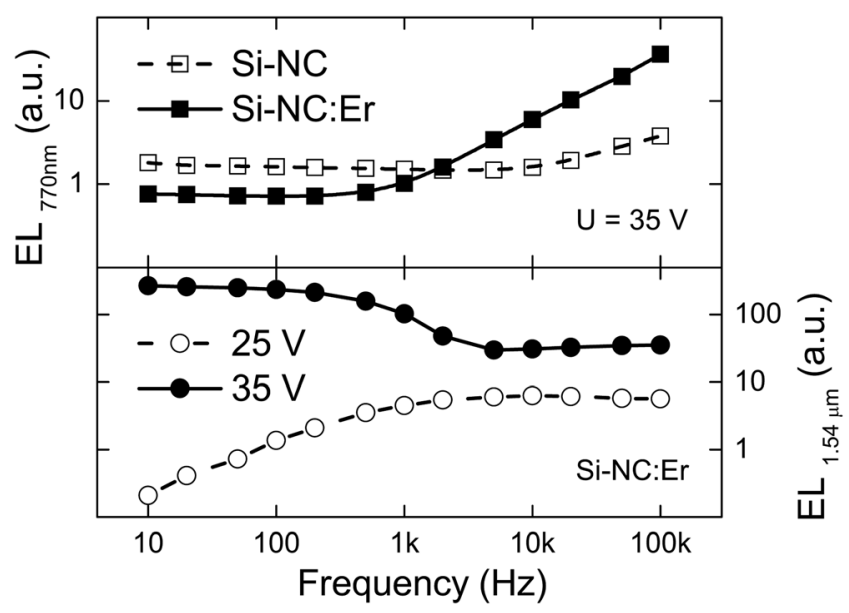

FIG. 5. (top panel) Peak EL intensity at $770 \mathrm{~nm}$ as a function of bipolarpulse driving frequency with a fixed RMS voltage of $35 \mathrm{~V}$ for both Si-NC and Si-NC:Er LEDs. (Bottom panel) Integrated EL spectral intensity in a wavelength range bracketing $1.54 \mu \mathrm{m}$ as a function of bipolar-pulse driving frequency at a fixed RMS voltage of 25 and $35 \mathrm{~V}$ for the Si-NC:Er LEDs. The meaning of the different symbols is stated in the figure legend. 
EL intensity decreases (increases) for high (low) bias. In a frequency range $f \gg 1 \mathrm{kHz}$, which we name a moderate frequency range, it changes only slightly for both the high and low bias. This behavior is accompanied by an increase of the peak EL intensity at $770 \mathrm{~nm}$ (Fig. 5, top panel; high bias). It should be noted that the onset frequency of this increase is around $800 \mathrm{~Hz}$ unlike the undoped Si-NC LED where the EL starts to significantly increase above $10 \mathrm{kHz}$. The EL decay lifetime of Si-NCs is around $5 \mu$ s (measured by both PL and EL), which corresponds to a frequency of $200 \mathrm{kHz}$. These high frequencies are not available with our instruments and, so that, the lifetime of Si-NCs does not limit the EL intensity in the studied frequency range.

The frequency dependence of the EL intensity is reflected in evident changes of the Si-NC:Er LED spectral characteristics, which are shown in Fig. 6 for high bias only. The multiple Er emission peaks weaken, including the emission at $1.54 \mu \mathrm{m}$ (Fig. 5), and the peaks at 550, 660, and 850 $\mathrm{nm}$ disappear at the moderate frequencies. The Si-NC emission peak emerges. We attribute these changes to a change in the dominant excitation mechanism of Er ions: from electron impact to the energy transfer between Si-NCs and Er ions. Under the pulsed excitation scheme, sequential injection of electrons and holes into $\mathrm{Si}-\mathrm{NCs}$ takes place at the bias transitions. ${ }^{9}$ More efficient injection into Si-NCs at high injection frequencies (more bias transitions for a same time period) provides additional indirect Er excitation by means of the energy transfer at the bias transitions. This explains an increase of the Er emission at $1.54 \mu \mathrm{m}$ with injection frequency at low voltages (there is no emission by impact), for example $25 \mathrm{~V}$ shown in Fig. 5. Note that optical excitation experiments show that only $\sim 1 \%$ of the total Er population is coupled to the Si-NCs. ${ }^{29}$ Therefore, if we assume that this holds true also for electrical excitation, it is this $1 \%$ of Er ions which shows an increased excitation due to a better energy transfer from the $\mathrm{Si}-\mathrm{NCs}$. If the injection frequency is smaller than the Er emission rate of about $800 \mathrm{~Hz}$, both Si$\mathrm{NCs}$ and $\mathrm{Er}$ are in relaxed states and follow the frequency. If the excitation frequency is higher than the Er emission rate (while still smaller than the Si-NC emission rate of 200 $\mathrm{kHz}$ ), Er stays in an excited state and further excitation is

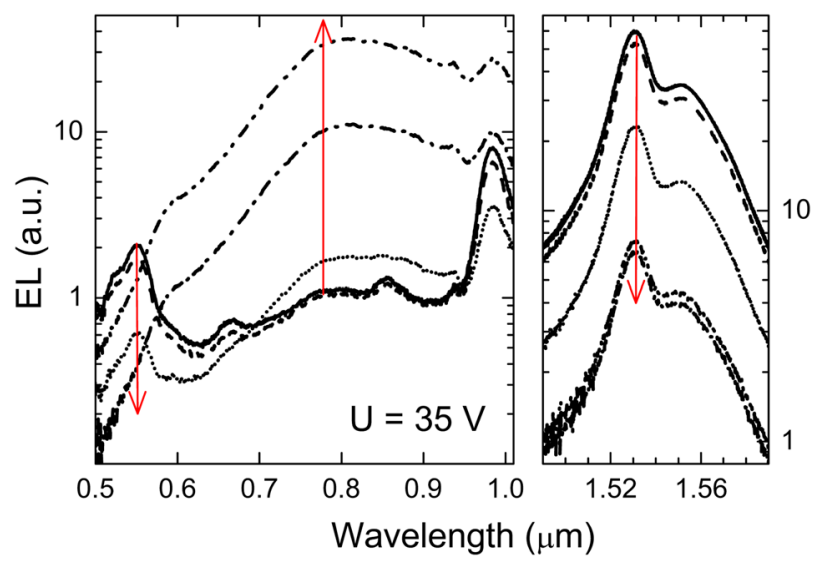

FIG. 6. The EL spectra at 10, 100, $1 \mathrm{k}, 10 \mathrm{k}$, and $50 \mathrm{kHz}$ for the Si-NC:Er LEDs shown in Fig. 5 for a fixed RMS voltage of 35 V. The ascending frequency order is indicated by the arrows.
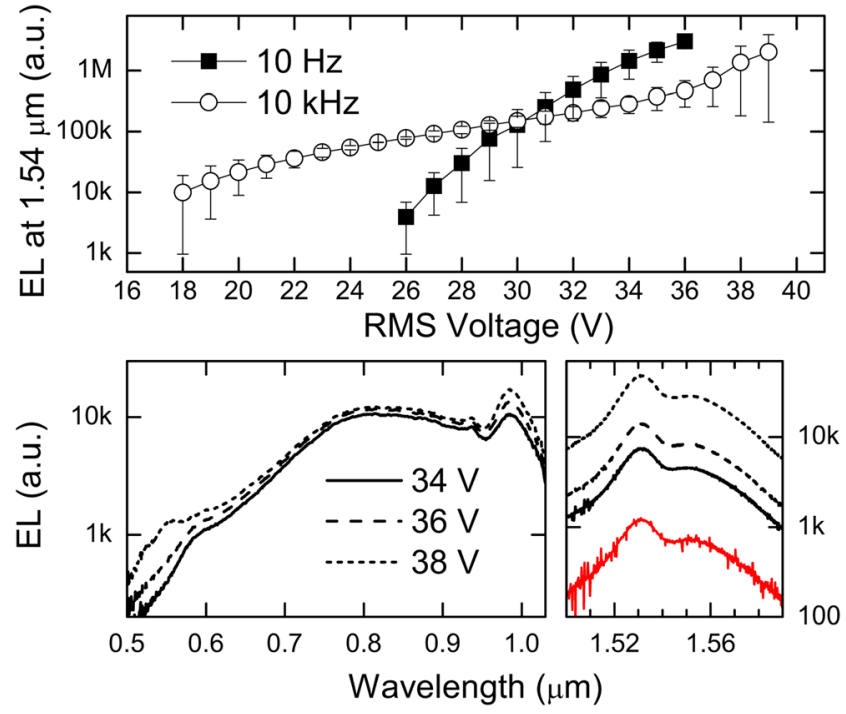

FIG. 7. EL intensity at $1.54 \mu \mathrm{m}$ as a function of RMS voltage under bipolar pulsed electrical excitation for two driving frequencies $10 \mathrm{~Hz}$ (squares) and $10 \mathrm{kHz}$ (circles). The low panels show EL spectra at 34, 36, and $38 \mathrm{~V}_{\mathrm{RMS}}$ at $10 \mathrm{kHz}$. Notice an appearance of the Er emission band at $550 \mathrm{~nm}$ at $38 \mathrm{~V}$. The lowest red line at the right $-\mathrm{EL}$ spectrum at $30 \mathrm{~V}_{\mathrm{RMS}}$ at $10 \mathrm{~Hz}$. No light emission was detected in the visible region at $30 \mathrm{~V}_{\mathrm{RMS}}$ at $10 \mathrm{~Hz}$.

limited by nonradiative processes. It should be noted that intensity of Er emission due to impact excitation decreases by a factor of 2 when the driving frequency increases from $10 \mathrm{~Hz}$ to $100 \mathrm{kHz}$. This follows from a measurement of $\mathrm{Er}$ emission intensity at $1.54 \mu \mathrm{m}$ versus driving frequency of high-voltage forward bias pulsed excitation, which turns the LED on and off. This decrease however cannot account for the decrease in EL intensity shown in Fig. 5 (bottom panel, full symbols) which is due to the bipolar pulsed excitation.

The fact that the energy transfer between Si-NCs and Er-ions becomes a dominant Er excitation mechanism at the moderate frequency range is further supported by the voltage dependence of EL intensity at $1.54 \mu \mathrm{m}$ shown in Fig. 7. The voltage dependence is stronger for low than for moderate injection frequencies. It is important that the EL signal in the moderate frequency range is observed at lower driving voltages than in DC or low frequency excitation, namely the onset of EL was measured at around $18 \mathrm{~V}_{\mathrm{RMS}}$ (Fig. 7). Beyond this value, charge trapping in the oxide arrests EL. The energy transfer remains the main excitation mechanism of Er up to about $36 \mathrm{~V}_{\mathrm{RMS}}$ at moderate frequencies. This is supported by the EL spectra shown in Fig. 7. There are no

TABLE I. Er excitation mechanisms. Low frequency stands for driving frequencies lower than the Er emission rate, moderate frequency — frequencies higher than the Er emission rate, but lower than the EL decay rate of SiNCs.

\begin{tabular}{lcc}
\hline \hline & $\begin{array}{c}\text { Low frequency, } \\
f<\sim 1 \mathrm{kHz}\end{array}$ & $\begin{array}{c}\text { Moderate frequency, } \\
1 \mathrm{kHz} \ll f<200 \mathrm{kHz}\end{array}$ \\
\hline $\begin{array}{l}\text { Low voltages, } \mathrm{U}<25 \mathrm{~V} \\
\text { Moderate voltages, }\end{array}$ & N/A & Transfer \\
$25 \mathrm{~V}<\mathrm{U}<36 \mathrm{~V}$ & Transfer and Impact & Transfer \\
High voltages, $\mathrm{U}>36 \mathrm{~V}$ & Impact & Impact \\
\hline
\end{tabular}


Er-related peaks at wavelengths below $980 \mathrm{~nm}$ at low-tomoderate voltages, while an Er peak centered at around 550 $\mathrm{nm}$ is observed at $38 \mathrm{~V}_{\mathrm{RMS}}$. At high voltages, ca. $36 \mathrm{~V}_{\mathrm{RMS}}$, and moderate frequencies, the EL emission is dominated by impact. Table I summarizes the obtained results in a broad range of excitation frequencies and voltages in terms of a dominant excitation mechanism of Er-ions under electrical pumping.

\section{CONCLUSIONS}

We have observed a change in the Er excitation mechanism under pulsed electrical injection and studied its dependence on the injection frequency and voltage pulse amplitude. We ascribe Er emission at high voltages (for both DC and pulsed injection) to the impact excitation by high energy electrons while Er emission is due to the nonradiative energy transfer from Si-NCs at low voltages. The Er emission at the low voltages becomes accessible only at moderate injection frequencies which are larger than the inverse of Er emission lifetime of a few milliseconds. This behavior is quite general since we observed similar trends (Table I) on a number of other Si-NC:Er LEDs with various structural parameters. For clarity, here we discussed a single kind of LED.

\section{ACKNOWLEDGMENTS}

This work was supported by EC through the project ICT-FP7-224312 HELIOS and by Italy-Spain integrated actions. D. N.-U. thanks the financial support of AGAUR through the Beatriu de Pinòs program. We acknowledge the contribution from Nicola Daldosso at an early stage of the work and Eveline Rigo for the photographs of the LEDs.

${ }^{1}$ O. Jambois, F. Gourbilleau, A. Kenyon, J. Montserrat, R. Rizk, and B. Garrido, Opt. Express 18, 2230 (2010).

${ }^{2}$ J. D. B. Bradley and M. Pollnau, Laser Photonics Rev. 5, 368 (2011).

${ }^{3}$ M. E. Castagna, S. Coffa, M. Monaco, L. Caristia, A. Messina, R. Mangano, and C. Bongiorno, Physica E (Amsterdam) 16, 547 (2003).

${ }^{4}$ F. Iacona, D. Pacifici, A. Irrera, M. Miritello, G. Franzo, F. Priolo, D. Sanfilippo, G. Di Stefano, and P. G. Fallica, Appl. Phys. Lett. 81, 3242 (2002).

${ }^{5}$ A. Muscara, M. E. Castagna, S. Leonardi, and S. Coffa, IEEE J. Quantum Electron. 47, 1362 (2011)

${ }^{6}$ G. Franzo, V. Vinciguerra, and F. Priolo, Appl. Phys. A: Mater. Sci. Process. 69, 3 (1999).
${ }^{7}$ I. Izeddin, D. Timmerman, T. Gregorkiewicz, A. S. Moskalenko, A. A. Prokofiev, I. N. Yassievich, and M. Fujii, Phys. Rev. B 78, 035327 (2008).

${ }^{8}$ A. Pitanti, D. Navarro-Urrios, N. Prtljaga, N. Daldosso, F. Gourbilleau, R. Rizk, B. Garrido, and L. Pavesi, J. Appl. Phys. 108, 053518 (2010).

${ }^{9}$ R. J. Walters, G. I. Bourianoff, and H. A. Atwater, Nature Mater. 4, 143 (2005).

${ }^{10}$ M. Peralvarez, C. Garcia, M. Lopez, B. Garrido, J. Barreto, C. Dominguez, and J. A. Rodriguez, Appl. Phys. Lett. 89, 051112 (2006).

${ }^{11}$ F. Priolo, C. D. Presti, G. Franzo, A. Irrera, I. Crupi, F. Iacona, G. Di Stefano, A. Piana, D. Sanfilippo, and P. G. Fallica, Phys. Rev. B 73, 113302 (2006).

${ }^{12}$ G. M. Miller, R. M. Briggs, and H. A. Atwater, J. Appl. Phys. 108, 063109 (2010).

${ }^{13}$ N. Prtljaga, D. Navarro-Urrios, A. Marconi, A. Anopchenko, J.-P. Colonna, F. Milesi, N. Daldosso, O. Jambois, B. Garrido, J.-M. Fedeli, and L. Pavesi, Opt. Mater. 33, 1083 (2011).

${ }^{14}$ F. Lenz, A. Hryciw, R. DeCorby, and A. Meldrum, Appl. Phys. Lett. 95, 091909 (2009).

${ }^{15}$ S. Cueff, C. Labbe, B. Dierre, F. Fabbri, T. Sekiguchi, X. Portier, and R. Rizk, J. Appl. Phys. 108, 113504 (2010).

${ }^{16}$ J. M. Sun, W. Skorupa, T. Dekorsy, M. Helm, and A. N. Nazarov, Opt. Mater. 27, 1050 (2005).

${ }^{17}$ O. Jambois, J. M. Ramirez, Y. Berencen, D. Navarro-Urrios, A. Anopchenko, A. Marconi, N. Prtljaga, A. Tengattini, P. Pellegrino, N. Daldosso, L. Pavesi, J.-P. Colonna, J.-M. Fedeli, and B. Garrido, J. Phys. D: Appl. Phys. 45, 045103 (2012).

${ }^{18}$ O. Jambois, Y. Berencen, K. Hijazi, M. Wojdak, A. J. Kenyon, F. Gourbilleau, R. Rizk, and B. Garrido, J. Appl. Phys. 106, 063526 (2009).

${ }^{19}$ L. Palmetshofer, Y. Suprun-Belevich, and M. Stepikhova, Nucl. Instrum. Methods Phys. Res. B 127, 479 (1997).

${ }^{20}$ D. Konig, M. Rennau, and M. Henker, Solid-State Electron. 51, 650 (2007).

${ }^{21}$ A. Nazarov, J. M. Sun, W. Skorupa, R. A. Yankov, I. N. Osiyuk, I. P. Tjagulskii, V. S. Lysenko, and T. Gebel, Appl. Phys. Lett. 86, 151914 (2005).

${ }^{22}$ T. Z. Lu, M. Alexe, R. Scholz, V. Talalaev, R. J. Zhang, and M. Zacharias, J. Appl. Phys. 100, 014310 (2006).

${ }^{23}$ B. Garrido, C. Garcia, S. Y. Seo, P. Pellegrino, D. Navarro-Urrios, N. Daldosso, L. Pavesi, F. Gourbilleau, and R. Rizk, Phys. Rev. B 76, 245308 (2007).

${ }^{24}$ P. M. Fauchet, Silicon Photonics 94, 177 (2004).

${ }^{25}$ N. Daldosso and L. Pavesi, Laser Photonics Rev. 3, 508 (2009).

${ }^{26}$ J. M. Ramírez, O. Jambois, Y. Berencén, D. Navarro-Urrios, A. Anopchenko, A. Marconi, N. Prtljaga, N. Daldosso, L. Pavesi, J.-P. Colonna, J. M. Fedeli, and B. Garrido, "Polarization strategies to improve the emission of Si-based light sources emitting at $1.55 \mu \mathrm{m}$ " Mater. Sci. Eng. B (to be published)

${ }^{27}$ S. Wang, A. Eckau, E. Neufeld, R. Carius, and C. Buchal, Appl. Phys. Lett. 71, 2824 (1997).

${ }^{28}$ G. Seguini, S. Schamm-Chardon, P. Pellegrino, and M. Perego, Appl. Phys. Lett. 99, 082107 (2011)

${ }^{29}$ N. Prtljaga, D. Navarro-Urrios, A. Tengattini, A. Marconi, A. Anopchenko, F. Ferrarese Lupi, O. Jambois, J. M. Ramirez, Y. Berencen, B. Garrido, F. Milesi, J.-P. Colonna, J.-M. Fedeli, and L. Pavesi (unpublished). 